\title{
The Role of National Media Programs in the Prevention of Childhood
}

\section{Cancer}

\author{
Mohammad Kajiyazdi ${ }^{1}$, Nayereh Jesmani ${ }^{2}$ and Kambiz Eftekhari (ii ${ }^{3,4,{ }^{*}}$ \\ ${ }^{1}$ Pediatric Hematology and Oncology Department, Bahrami Children's Hospital, Tehran University of Medical Sciences, Tehran, Iran \\ ${ }^{2}$ Azad University of Tehran, Tehran, Iran \\ ${ }^{3}$ Pediatric Gastroenterology and Hepatology Research Center, Tehran University of Medical Sciences, Tehran, Iran \\ ${ }^{4}$ Pediatric Department, Bahrami Children's Hospital, Tehran University of Medical Sciences, Tehran, Iran \\ "Corresponding author: Pediatric Department, Bahrami Children’s Hospital, Tehran University of Medical Sciences, Tehran, Iran. Email: dr_k_eftekhary@yahoo.com
}

Received 2020 October 27; Revised 2020 November 22; Accepted 2020 November 24.

\begin{abstract}
Background: In the contemporary century, the media has played a significant role in educating communities.

Objectives: The purpose of this research is to investigate the role of national media programs in the prevention of childhood cancer. Methods: Participants in this research study included ordinary people, parents of children with cancer, and pediatric oncologists. The study was performed for six months (2017) in Bahrami and Mahak hospitals. Participants were asked questions about the role of the media in the prevention of childhood cancer, and their answers were recorded in a questionnaire.

Results: The results showed that, while ordinary people evaluate the role of national media programs, their simplicity and comprehensibility in the prevention of childhood cancer as desirable, parents of children with cancer consider this program's comprehensibility as low. Physicians also considered the influence of the contents of radio and television programs as low but their simplicity as desirable.

Conclusions: Our study confirmed the effectiveness of national media programs in the prevention of childhood cancer. The level of education of parents was effective in understanding media education in the prevention of childhood cancer.
\end{abstract}

Keywords: Cancer, Child, Media Programs, Prevention

\section{Background}

The role of the media in the contemporary century is becoming more prominent every day. Nowadays, the media is influencing societies deeply by discovering and applying new communication technologies. It has a significant and decisive impact on the orientation of public opinion. Only people or nations who have the needed power and ability to "refine waves" and "organize information" will be able to utilize the new age optimally (1). The media has given rise to thought, behavior, and culture and also monopolized the control of behavior and thought by transmitting the message and information to the recipients (2). Today, one of the important roles of the media is education. This tool has a great contribution in publishing and teaching the correct patterns of behavior, cognition, and prevention of diseases in society. Our attitude towards illness and the sick person is influenced by media education. The importance of this issue should be considered in health education programs and advertising cam- paigns, media support, and even health education activities. Therefore, health and media researchers should pay attention to the priorities, statements in the media, and the audience's reaction to media messages (3). Cancer is the second leading cause of death after cardiovascular diseases (4). In the coming years, the prevalence of the disease will rise; therefore, it can be a potential health problem. Cancers are a costly disease that imposes huge costs on the health budgets of countries and their families each year. Therefore, research on cancer is essential to improve the quality of prevention, effective and cheaper treatments, and rehabilitation. In general, $85 \%$ of children with cancer recover, but in our country, only $50 \%$ of them are treated, which indicates the importance of this issue. In this regard, new communication technologies and video and audio media have a very important role. Understanding the structure of mass media and how effective it is for the health of society is very important. 


\section{Objectives}

This study aimed to assess the role of national media in the prevention of childhood cancer.

\section{Methods}

Participants in this research study included ordinary people (as the non-cancer group), parents of children with cancer (as the cancer group), and pediatric oncologists (as the physician group). The sampling method in the study was stratified, and the sampling method within classes was simple random sampling or convenience sampling. The sample size of the cancer group, non-cancer group, and physician group was calculated 356 (according to the Cochran's formula), 384 (according to Morgan's table), and 30 (according to the Cochran's formula), respectively. Simple random sampling was used to select cancer and noncancer groups. The convenience sampling was used to select the group of physicians (due to the specialization of the subject). This study was performed on the families of cancer patients referring to Bahrami and Mahak hospitals from spring to summer of 2017. The control group was selected from the general population and the physician group from the staff of Bahrami Children's Hospital. Initially, written informed consent was obtained from the participants. The demographic data were comprised of gender, age, and occupation. Information about the following questions was obtained from the participants, and the results were recorded in separate questionnaires.

1) Do you watch medical, educational, and health television programs?

2) Do medical, educational, and health television programs affect the prevention of childhood cancer?

3) Do you listen to medical, educational, and health radio programs?

4) Do medical, educational, and health radio programs improve the prevention of childhood cancer?

5) Are the medical, educational, and health television programs comprehensible?

6) Are the medical, educational, and health radio programs comprehensible?

The validity of the questionnaire was confirmed by the opinions of physicians, media experts, and medical staff. The questionnaire reliability regarding the sample of noncancer family and those who have cancer family was acceptable (0.658) and good (0.819). However, the questionnaire reliability of the physicians' sample could not be calculated due to the small sample. In this research, quantitative data were used as a questionnaire technique. Written questionnaires in three groups were presented to "people who have a child with cancer", "non-cancer people”, and "pediatric cancer specialists". The Chi-square test was used to analyze the nominal qualitative data. Finally, the data were processed with SPSS software (version 22), and statistical methods and tests were used to investigate research questions according to the level of measurement of variables.

\section{Results}

Four hundred and fifty-three (453) people enrolled in the study and completed the questionnaire that included 365 non-cancer families, 81 cancer families, and seven physicians. One hundred and eighteen (33.1\%) participants in the study were male. The mean age of them was $30 \pm 10$ years (20 - 61 years). In the non-cancer and cancer group, 22.3\% and $72.3 \%$ had a diploma or lower education, respectively. In Table 1, the results of the questionnaire are summarized. A total of 343 participants watched medical, educational, and health television programs in three groups. The majority of these cases, which included $47.2 \%(n=162)$, believed that these programs are moderately effective in preventing cancer. Most of them stated that these programs had simplicity and comprehensibility at a moderate level. A total of 120 participants listened to medical, educational, and health radio programs in all groups. The majority of cases in the non-cancer and physician groups believed that these programs are moderately effective in preventing cancer, unlike the cancer group (Table 2). There was a significant difference between the groups in terms of the effectiveness of the radio and television medical programs in preventing cancer in children (P-value $=0.001$ ). Many stated that these programs had simplicity and comprehensibility at a moderate level (Table 3). There was a significant difference between the groups in terms of simplicity and comprehensibility of the radio and television programs $(\mathrm{P}$-value $=0.001)$.

\section{Discussion}

According to our results, television programs have a favorable impact on preventing childhood cancer from the viewpoint of the non-cancer group, unlike other groups. To explain this, it can be said that the non-cancer group did not pay much attention to these programs due to the lack of need to obtain information about cancer. The non-cancer group measured the impact of radio medicine programs on the prevention of childhood cancer on the 


\begin{tabular}{|c|c|c|c|c|c|c|c|c|c|c|}
\hline \multicolumn{5}{|l|}{ Questioner } & \multicolumn{2}{|c|}{ Non-cancer Families } & \multicolumn{2}{|c|}{ Cancer Families } & \multicolumn{2}{|c|}{ Physicians } \\
\hline \multicolumn{5}{|c|}{ Do you watch medical, educational, and health television programs? } & \multicolumn{2}{|c|}{296 (83.1) Positive } & \multicolumn{2}{|c|}{42 (51.9) Positive } & \multicolumn{2}{|c|}{5 (71.4) Positive } \\
\hline \multicolumn{5}{|c|}{$\begin{array}{l}\text { Do medical, educational, and health television programs affect the prevention } \\
\text { of childhood cancer? }\end{array}$} & \multicolumn{2}{|c|}{141 (40.9) Moderate } & \multicolumn{2}{|c|}{24 (33.3) Very little } & \multicolumn{2}{|c|}{ 3(42.9) Moderate } \\
\hline \multicolumn{5}{|c|}{ Do you listen to medical, educational, and health radio programs? } & \multicolumn{2}{|c|}{90 (25.8) Positive } & \multicolumn{2}{|c|}{26 (32.9) Positive } & \multicolumn{2}{|c|}{4 (57.1) Positive } \\
\hline \multicolumn{5}{|c|}{$\begin{array}{l}\text { Do medical, educational, and health radio programs affect the prevention of } \\
\text { childhood cancer? }\end{array}$} & \multicolumn{2}{|c|}{115 (35) Moderate } & \multicolumn{2}{|c|}{26 (49.1) Very little } & \multicolumn{2}{|c|}{ 3(42.9) Moderate very little } \\
\hline \multicolumn{5}{|c|}{ Are the medical, educational, and health television programs comprehensible? } & \multicolumn{2}{|c|}{163 (48.4) Moderate } & \multicolumn{2}{|c|}{29 (36.7) Moderate } & \multicolumn{2}{|c|}{4 (57.1) High } \\
\hline \multicolumn{5}{|c|}{ Are the medical, educational, and health radio programs comprehensible? } & \multicolumn{2}{|c|}{156 (49.2) Moderate } & \multicolumn{2}{|c|}{ 21(38.2) Little } & \multicolumn{2}{|c|}{ 3(42.9) Moderate high } \\
\hline Study Group & $\begin{array}{l}\text { The Effect of } \\
\text { Programs }\end{array}$ & Very High & High & Interm & liate & Low & Very Low & & o Answer & Total \\
\hline \multirow{2}{*}{$\begin{array}{l}\text { Non-cancer } \\
\text { families }\end{array}$} & Television & 21 & 76 & 14 & & 72 & 35 & & 20 & 365 \\
\hline & Radio & 14 & 63 & 115 & & 85 & 52 & & 36 & 365 \\
\hline \multirow{2}{*}{ Cancer families } & Television & 10 & 8 & 18 & & 12 & 24 & & 9 & 81 \\
\hline & Radio & 2 & 10 & 7 & & 8 & 26 & & 28 & 81 \\
\hline \multirow{2}{*}{ Physicians } & Television & 0 & 1 & 3 & & 1 & 2 & & 0 & 7 \\
\hline & Radio & 0 & 0 & 3 & & 1 & 3 & & 0 & 7 \\
\hline
\end{tabular}

${ }^{\mathrm{a}}$ There was a significant difference between the groups in terms of the effectiveness of the radio and television medical programs in preventing cancer in children $($ P-value $=0.001)$.

\begin{tabular}{|c|c|c|c|c|c|c|c|}
\hline Study Group & $\begin{array}{l}\text { The Simplicity } \\
\text { of Programs }\end{array}$ & Very High & High & Intermediate & Low & Very Low & Total \\
\hline \multirow{2}{*}{ Non-cancer families } & Television & 15 & 82 & 89 & 7 & 1 & 194 \\
\hline & Radio & 3 & 39 & 39 & 7 & 1 & 89 \\
\hline \multirow{2}{*}{ Cancer families } & Television & 2 & 12 & 14 & 14 & 0 & 42 \\
\hline & Radio & 0 & 6 & 6 & 12 & 2 & 26 \\
\hline \multirow{2}{*}{ Physicians } & Television & 0 & 3 & 2 & 0 & 0 & 5 \\
\hline & Radio & 0 & 2 & 2 & 0 & 0 & 4 \\
\hline
\end{tabular}

${ }^{\mathrm{a}}$ There was a significant difference between the groups in terms of simplicity and comprehensibility of the radio and television programs $(\mathrm{P}$-value $=0.001)$.

medium level, while other groups have found the effect to be very poor. The non-cancer and physician groups assessed national media programs (TV and radio) as simple and understandable, while the cancer group described television programs as simple and desirable, but radio programs as weak. This suggests that television messages may be simpler and more comprehensible because they are audio-visual, while radio messages are less comprehensible because they are audible and one-dimensional. Many people in the non-cancer and physician groups had higher education, so they had no problem understanding medical programs, but the cancer group often had a diploma or lower degree, so they had difficulties in understanding these programs.

According to research in the scientific literature, the role of national media in the prevention of childhood cancer has not been studied. However, various studies have been conducted on the role of media in the education and prevention of AIDS and the education of children. A study by Sadeghi et al. showed that radio and television have had a greater impact on educating students about AIDS (5). The results of a study by Razavi et al. (6) showed that the mass media changed people's attitudes towards public sports but did not have a significant effect. In September 2002, Parker et al. (7) designed a Strategic Communication Campaign to promote education and awareness of 
the importance of proper nutrition, exercise, and a healthy lifestyle to prevent cancer, early detection of cancer, and increase awareness and understanding of the five major cancers among Georgians. The results of the research showed that the mass media have a good influence on serving the interests of society, especially public health. Bell et al. (8) showed that media advertising has been effective in preventing childhood obesity by raising awareness about healthy eating and proper physical activity.

The strengths of this study were as follows: the first study was on the effectiveness of public media on the prevention of childhood cancer. Surveys were conducted in all age groups, sex, and level of education. The effectiveness of the media was evaluated in different separate groups. The limitations of this study included difficulty in communicating with the cancer group and impossibility of access to the cancer group.

\subsection{Conclusions}

The present study confirmed a relationship between the target groups and the impact of national media programs. In addition, parental education, especially for mothers, appears to be effective in preventing childhood cancer.

\section{Footnotes}

Authors' Contribution: Study concept and design: Mohammad Kajiyazdi, Nayereh Jesmani, and Kambiz Eftekhari. Acquisition of data: Mohammad Kajiyazdi, Nayereh Jesmani, and Kambiz Eftekhari. Analysis and interpretation of data: Mohammad Kajiyazdi and Kambiz
Eftekhari. Drafting of the manuscript: Mohammad Kajiyazdi and Kambiz Eftekhari. Critical revision: Mohammad Kajiyazdi, Nayereh Jesmani, and Kambiz Eftekhari. All authors approved the final version of the paper.

Conflict of Interests: The authors have no conflicts of interest relevant to this article.

Ethical Approval: This study was approved by the Ethics Committee of the Tehran University of Medical Sciences.

Funding/Support: No external funding was secured for this study.

\section{References}

1. Burger A. [Media Research Methods Translated by Mohammad Hafezati]. Tehran: Office of Media Studies and Planning (Ministry of Culture and Islamic Guidance); 1995. Persain.

2. Yavari Vasaq M. [The Role of Media in Political and Social Awareness] Tehran: Islamic Research Center. Islamic Republic of Iran Broadcasting; 2012. Persian.

3. Pourkarimi J. [Media Advertising and Audience Attitude Change].JRes Eval. 2000. Persian.

4. Heron M. Deaths: Leading Causes for 2017. Natl Vital Stat Rep. 2019;68(6):1-77. [PubMed: 32501203].

5. Sadeghi Lavasani Nia N, Sedghi J, Ahmadi H, Khodabakhsh R. The Role of Communication Media in Awareness and Prevention of AIDS among Students. Media Stud. 2013;8(21):123-36.

6. Razavi SMH, Dousti M, Ghasemi SM. The Role Of Mass Media On People's Attitude Towards Sport For All With A Focus On Physical Fitness (A Case Study: Babolsar City). Appl Res Sport Manag. 2015;4(13).

7. Parker DM. Georgia's Cancer Awareness and Education Campaign combining public health models and private sector communications strategies. Prev Chronic Dis. 2004;1(3). A09. [PubMed: 15670430]. [PubMed Central: PMC1253474].

8. Bell AC, Wolfenden L, Sutherland R, Coggan L, Young K, Fitzgerald M, et al. Harnessing the power of advertising to prevent childhood obesity. Int J Behav Nutr Phys Act. 2013;10:114. doi: 10.1186/1479-5868-10-114. [PubMed: 24090174]. [PubMed Central: PMC3852185]. 